\title{
Missed Doses of Venous Thromboembolism (VTE) Prophylaxis at Community Hospitals: Cause for Alarm
}

\author{
Brandyn D. Lau, MPH, CPH 1,2,3,4, Michael B. Streiff, MD, FACP 3,5,6, Peggy S. Kraus, PharmD, CACP', \\ Deborah B. Hobson, BSN $N^{3,8}$, Dauryne L. Shaffer, MSN ${ }^{8}$, Jonathan K. Aboagye, MBChB, MPH ${ }^{8}$, \\ Peter J. Pronovost, MD, PhD $3,4,9$, and Elliott R. Haut, MD, PhD, FACS $3,4,8,9,10$
}

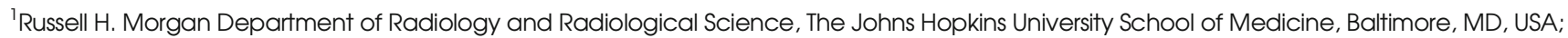
${ }^{2}$ Division of Health Sciences Informatics, The Johns Hopkins University School of Medicine, Baltimore, MD, USA; ${ }^{3}$ The Armstrong Institute for Patient Safety and Quality, Johns Hopkins Medicine, Baltimore, MD, USA; ${ }^{4}$ Department of Health Policy and Management, Johns Hopkins Bloomberg School of Public Health, Baltimore, MD, USA; ${ }^{5}$ Department of Medicine, The Johns Hopkins University School of Medicine, Baltimore, USA; ${ }^{6}$ Department of Pathology, The Johns Hopkins University School of Medicine, Baltimore, MD, USA; ${ }^{7}$ Department of Pharmacy, The Johns Hopkins Hospital, Baltimore, MD, USA; ${ }^{8}$ Department of Surgery, The Johns Hopkins University School of Medicine, Baltimore, MD, USA; ${ }^{9}$ Department of Anesthesiology \& Critical Care Medicine, The Johns Hopkins University School of Medicine, Baltimore, MD, USA; ${ }^{10}$ Department of Emergency Medicine, The Johns Hopkins University School of Medicine, Baltimore, MD, USA.

KEY WORDS: venous thromboembolism; quality improvement; safety. J Gen Intern Med 33(1):19-20

DOI: $10.1007 / \mathrm{s} 11606-017-4203-y$

(c) Society of General Internal Medicine 2017

\section{INTRODUCTION}

Venous thromboembolism (VTE) is a leading cause of potentially preventable harm. Randomized controlled trials have demonstrated that VTE prophylaxis, when administered completely, significantly reduces the risk for deep vein thrombosis, pulmonary embolism (PE), and fatal PE. ${ }^{1}$

Numerous interventions have been successful in improving the prescription of VTE prophylaxis. ${ }^{2}$ Unfortunately, few interventions have ensured that prescribed VTE prophylaxis is actually administered. One study from a large academic hospital reported that $10 \%$ of VTE prophylaxis doses were not administered. $^{3}$ A larger study in another academic hospital found that $12 \%$ of VTE prophylaxis doses were not administered, where $40.6 \%$ of all hospitalized patients and $46.2 \%$ of all medically ill patients missed $\geq 1$ dose. $^{4}$

This small but growing body of evidence has come entirely from academic hospitals, and thus provides no insight into practice at community hospitals, which account for the vast majority of hospitals and hospital admissions. This study compared VTE prophylaxis medication non-administration between a major quaternary academic university hospital and three community hospitals within a large health system.

\section{METHODS}

In this retrospective study, we included four hospitals within the Johns Hopkins Health System: The Johns Hopkins Hospital (JHH; academic), Howard County General Hospital (community), Sibley Memorial Hospital (community), and Suburban Hospital (community). We queried the electronic medication administration record system for each hospital from January 1

Published online October 17, 2017 through December 31, 2015, to identify patients who were prescribed pharmacological VTE prophylaxis, including unfractionated heparin $(5000 \mathrm{U} \mathrm{q} 12 \mathrm{~h} / \mathrm{q} 8 \mathrm{~h}$ or $7500 \mathrm{U}$ $\mathrm{q} 12 \mathrm{~h} / \mathrm{q} 8 \mathrm{~h}$ ), enoxaparin ( $30 \mathrm{mg} \mathrm{q} 12 \mathrm{~h} / \mathrm{q} 24 \mathrm{~h}$ or $40 \mathrm{mg}$ $\mathrm{q} 12 \mathrm{~h} / \mathrm{q} 24 \mathrm{~h})$, fondaparinux (2.5 $\mathrm{mg} \mathrm{q} 24 \mathrm{~h})$, and dalteparin (5000 U q24h). Nurses must document every dose as either administered or not administered for each scheduled administration time. We calculated the proportion of overall doses not administered and the proportion of patients who missed $\geq 1$ dose, by individual hospital and hospital type. Proportions were compared using chi-square tests. This study was approved by the Johns Hopkins Medicine Institutional Review Board.

\section{RESULTS}

At JHH, 10.9\% $(33,971 / 311,294)$ of VTE prophylaxis medication doses were not administered. Overall, non-administration was significantly higher $13.6 \%(21,580 / 158,938, p<0.001$, Table 1) at the three community hospitals. The proportion of doses not administered varied significantly, from $10.9 \%$ to $15.0 \%$ ( $p<0.001)$, among community hospitals.

At JHH, $43.7 \%(10,795 / 24,709)$ of patients who were prescribed VTE prophylaxis medication missed $\geq 1$ dose. At the three community hospitals, a similar proportion of patients $(43.6 \%, 8002 / 18,355, p=0.852)$ missed $\geq 1$ dose. Among community hospitals, the proportion of patients who missed $\geq 1$ dose varied greatly, from $36.2 \%$ to $52.0 \%$ ( $p<0.001$, Table 1 ).

\section{DISCUSSION}

Non-administration of VTE prophylaxis medication is a pervasive problem in both academic and community hospitals, where $10-15 \%$ of doses are not administered. Nearly half of hospitalized patients missed $\geq 1$ dose. Overall, nonadministration was lowest at the academic hospital and significantly higher at the three community hospitals. 
Table 1 VTE Prophylaxis Non-Administration in a Large Health System from January 1 through December 31, 2015, by Hospital

\begin{tabular}{|c|c|c|c|c|c|}
\hline Hospital & Type & $\begin{array}{l}\text { VTE prophylaxis doses } \\
\text { not administered }\end{array}$ & $P$-value & Patients who missed $\geq 1$ dose & $P$-value \\
\hline The Johns Hopkins Hospital & Academic & $33,971 / 311,294(10.9 \%)$ & $<0.001 *$ & $10,795 / 24,709(43.7 \%)$ & $0.852 *$ \\
\hline Combined community hospitals & Community & $21,550 / 158,938(13.6 \%)$ & & $8002 / 18,355(43.6 \%)$ & \\
\hline Howard County General Hospital & Community & $11,952 / 79,544(15.0 \%)$ & $<0.001^{\dagger}$ & $3854 / 7406(52.0 \%)$ & $<0.001^{\dagger}$ \\
\hline Sibley Memorial Hospital & Community & $3367 / 31,003(10.9 \%)$ & & $1477 / 4076(36.2 \%)$ & \\
\hline Suburban Hospital & Community & $6231 / 48,391(12.9 \%)$ & & $2671 / 6873(38.9 \%)$ & \\
\hline
\end{tabular}

*Comparing combined community hospitals with academic hospital

${ }^{\dagger}$ Comparing across community hospitals

A study assessing nursing perceptions regarding VTE prophylaxis found that nurses frequently use their clinical decisionmaking skills to determine when patients might be of sufficiently low risk to justify omitting prescribed VTE prophylaxis. ${ }^{6}$ Higher VTE prophylaxis non-administration at community hospitals may be consistent with an anecdotal notion that patients in community hospitals are at lower risk for VTE. However, evidence suggests that missing doses of prophylaxis may lead to potentially preventable VTE among hospitalized patients. ${ }^{5}$ These findings underscore the importance of identifying patients who are at high risk both for developing VTE and for missing prophylaxis.

We were not able to categorize patients by specialty (i.e. surgery vs. medicine) from our dataset. However, it has been reported that medically ill patients miss significantly more doses than other hospitalized patients. ${ }^{4}$ Also, we did not report VTE outcomes, as this was beyond the scope of the current study. The association between non-administration of VTE prophylaxis and development of VTE would require accounting for specific patient-level risk factors that were not available, and information about the sequence of non-administration; reporting VTE outcomes alone would oversimplify the development of VTE.

These data support the need to promote efforts to reduce the incidence of VTE by improving administration of prescribed doses of VTE prophylaxis. All hospitals should monitor VTE prophylaxis medication administration practice, and adopt successful and sustainable interventions to improve these practices. While VTE prophylaxis prescription has historically been the focus, the next frontier of VTE prevention should focus on interventions to improve administration of prescribed VTE prophylaxis in all hospital settings.

\section{Acknowledgements:}

Contributors: We would like to thank Ann Hoffman, MBA, and Shamil Fayzullin for their assistance in obtaining medication administration data.

Corresponding Author: Brandyn D. Lau, MPH, CPH; Russell H. Morgan Department of Radiology and Radiological Science, The Johns Hopkins University School of Medicine, Baltimore, MD, USA (e-mail: blau2@jhmi.edu).

Funders: This project was supported by contract CE-12-11-4489 "Preventing Venous Thromboembolism: Empowering Patients and Enabling Patient-Centered Care via Health Information Technology" from The Patient-Centered Outcomes Research Institute (PCORI).

\section{Compliance with Ethical Standards:}

Conflict of Interest: Mr. Lau and Drs. Streiff and Haut are supported by a grant from the AHRQ (1RO1HS024547) entitled "Individualized Performance Feedback on Venous Thromboembolism Prevention Practice," a contract from PCORI entitled "Preventing Venous Thromboembolism (VTE): Engaging Patients to Reduce Preventable Harm from Missed/Refused Doses of VTE Prophylaxis," and a grant from the NIH/ NHLBI (R21HL129028) entitled "Analysis of the Impact of Missed Doses of Venous Thromboembolism Prophylaxis." Mr. Lau is supported by the Institute for Excellence in Education Berkheimer Faculty Education Scholar Grant and a contract (AD-1306-03980) from the PatientCentered Outcomes Research Institute (PCORI) entitled "Patient-Centered Approaches to Collect Sexual Orientation/Gender Identity Information in the Emergency Department." Ms. Hobson has given expert witness testimony in various medical malpractice cases. Dr. Pronovost reports consultancy fees from the Association for Professionals in Infection Control and Epidemiology, Inc.; grant or contract support from the Agency for Healthcare Research \& Quality, National Institutes of Health, Robert Wood Johnson Foundation, Patient-Centered Outcomes Research Institute, and The Commonwealth Fund; honoraria from various hospitals and the Leigh Bureau (Somerville, NJ); and royalties from his book, Safe Patients, Smart Hospitals. Dr. Streiff has received research funding from Portola and Janssen, consulted for Bio2 Medical, CSL Behring, Merck and Janssen Healthcare, and has given expert witness testimony in various medical malpractice cases. Dr. Haut is a paid consultant and speaker for the "Preventing Avoidable Venous Thromboembolism-Every Patient, Every Time" VHA/Vizient IMPERATIV ${ }^{\mathbb{B}}$ Advantage Performance Improvement Collaborative. Dr. Haut receives royalties from Lippincott, Williams \& Wilkins for the book Avoiding Common ICU Errors. Dr. Haut is a paid consultant and speaker for the Illinois Surgical Quality Improvement Collaborative (ISBIC). Dr. Haut was the paid author of a paper commissioned by the National Academies of Medicine titled "Military Trauma Care's Learning Health System: The Importance of Data Driven Decision Making," which was used to support the report titled A National Trauma Care System: Integrating Military and Civilian Trauma Systems to Achieve Zero Preventable Deaths After Injury. All other authors declare that they have no conflict of interest.

\section{REFERENCES}

1. Streiff MB, Lau BD. Thromboprophylaxis in nonsurgical patients. Hematology Am Soc Hematol Educ Program. 2012;2012:631-7.

2. Lau BD, Haut ER. Practices to prevent venous thromboembolism: a brief review. BMJ Qual Saf. 2014;23(3):187-95.

3. Fanikos J, Stevens LA, Labreche M, et al. Adherence to pharmacological thromboprophylaxis orders in hospitalized patients. Am J Med. 2010; 123(6):536-41

4. Shermock KM, Lau BD, Haut ER, et al. Patterns of non-administration of ordered doses of venous thromboembolism prophylaxis: Implications for novel intervention strategies. PLoS ONE. 2013;8(6):e66311.

5. Haut ER, Lau BD, Kraus PS, et al. Preventability of hospital-acquired venous thromboembolism. JAMA Surg. 2015;150(9):912.

6. Elder S, Hobson DB, Rand CS, et al. Hidden barriers to delivery of pharmacological venous thromboembolism prophylaxis: The role of nursing beliefs and practices. J Patient Saf. 2016;12(2):63-8. 